\title{
Homogeneous cooling state of a low-density granular flow
}

\author{
J. Javier Brey, M. J. Ruiz-Montero, and D. Cubero \\ Departamento de Física Teórica, Universidad de Sevilla, Apartado de Correos 1065, E-41080 Sevilla, Spain
}

(Received 12 June 1996)

\begin{abstract}
The homogeneous cooling state of a granular flow of smooth spherical particles described by the Boltzmann equation is investigated by means of the direct simulation Monte Carlo method. The velocity moments and also the velocity distribution function are obtained and compared with approximate analytical results derived recently. The accuracy of a Maxwell-Boltzmann approximation with a time-dependent temperature is discussed. Besides, the simulations show that the state of uniform density is unstable to long enough wavelength perturbations so that clusters and voids spontaneously form throughout the system. The instability has the characteristic features of the clustering instability which has been observed in molecular dynamics simulations of dense fluids and predicted by hydrodynamic models of granular flows. [S1063-651X(96)03710-5]

PACS number(s): 05.20.Dd, 47.50.+d, 47.20.-k
\end{abstract}

\section{INTRODUCTION}

Granular materials consist of macroscopic solid particles immersed in gas or liquid. When driven to rapid flows their behavior is dominated by the particle collisions and the interstitial fluid plays a negligible role in the flow mechanics. As an idealization, the granular flow can be considered as similar to a flowing fluid composed of the grains. In recent years, this analogy has been used to derive continuum equations for the evolution of the mass, momentum, and energy of rapid granular flows. This has led to the proposal of hydrodynamiclike equations analogous to the conventional Navier-Stokes equations for normal fluids [1]. Nevertheless, unlike usual fluids, the kinetic energy in granular systems is not conserved in collisions due to inelasticity and also to surface roughness of the grains. There is a conversion of kinetic energy into internal energy and the total kinetic energy of a granular flow tends to decrease in time. As a consequence, a term describing the dissipation of energy in collisions is included in the evolution equation for the energy.

A solid justification of the validity of a hydrodynamic description for granular flows can only be achieved by starting from a more fundamental description of the system. For ordinary fluids, the connection between a particle description and a continuous description is provided by kinetic theory. It is then not surprising that kinetic theory methods have been extended to the case of inelastic collisions between particles [2-6]. In the most idealized case, the grains are modeled by equal smooth spherical particles, whose interactions only affect the translational degrees of freedom. A more realistic description requires the consideration of rough particles [7-9] and also of particles of different sizes [10-12].

Application of kinetic theory methods implies, firstly, the derivation of a kinetic equation for the distribution function and, secondly, the solution of this equation. Although most of the kinetic equations proposed up to now for granular flows have been formulated by means of intuitive arguments, similar to those used in the heuristic derivations of the Boltzmann and Enskog equations [13], very recently the problem has been formulated at the level of a pseudoLiouville equation for inelastic spheres [6,14]. From this equation, the Bogoliubov-Born-Green-Kirkwood-Yvon
(BBGKY) hierarchy for the reduced distributions functions is derived, and, in the appropriate limits, extensions of the Boltzmann equation and the revised Enskog equation for dissipative dynamics are obtained.

Once the kinetic equation for the one-particle distribution function is known, the standard procedure to obtain hydrodynamic equations, with explicit expressions for the fluxes valid up to first order in the fields, is the Chapman-Enskog expansion [13]. Nevertheless, a difficulty arises during the application of this method to granular media as a consequence of dissipation in collisions. While the zeroth order distribution in the case of normal fluids is given by the local equilibrium distribution, the solution of the corresponding equation for inelastic collisions is not known. The equation is formally the same as the one describing the homogeneous granular state, whose time dependence occurs entirely through the energy field. In fact, this state plays the same role for fluids with inelastic collisions as the Maxwellian does for normal fluids. In the latter case, a local equilibrium generalization of the Maxwellian provides the reference state for the study of inhomogeneous states. A similar local cooling state exists for inelastic collisions, but the expression of the distribution function for this state is not known, even in the case of a dilute gas described by the (inelastic) Boltzmann equation. Then, what is usually done in existing kinetic theories of granular flows is to introduce some approximated expression for this reference single particle distribution function. On the basis of molecular dynamics results and qualitative reasoning, a Maxwellian is normally used. Corrections to it have been recently obtained by introducing expansions in Hermitian or Sonine polynomials, whose coefficients are determined in a consistent way $[9,14]$. It must be noticed that deviations from the Maxwellian, even if they are quantitatively small, can be very important from a physical and also a practical point of view, since they can even imply the presence of new contributions to the fluxes which are not present in normal fluids $[4,15]$.

Molecular dynamics studies [16-19] have clearly established that uniform cooling granular systems are unstable, and particles spontaneously tend to form clusters surrounded by regions of low density. This phenomenon has been referred to as "clustering instability" and has been explained 
using hydrodynamic linear stability theory [20]. Since the Boltzmann equation describes a low-density gas in which correlations between colliding particles are neglected, it is not clear a priori whether it also presents a clustering instability or, at least, some kind of inhomogeneous instability which can be considered as a precursor to clustering.

The direct Monte Carlo simulation method was developed by Bird twenty years ago to simulate the Boltzmann equation $[21,22]$. It allows quantitative study of most low-density gas flows, and can be easily adapted to the case of inelastic collisions between particles. The aim of this paper is to report some simulation results obtained for the homogeneous cooling state using this method. From the simulations it is possible to compute the one-particle distribution function over a quite wide range of velocities and, in particular, precise values for the fourth and sixth velocity moments are obtained. This provides a test for the approximated solutions to the Boltzmann equation discussed above. Also the stability of the homogeneous cooling state is investigated, showing that the Boltzmann equation presents an instability which has all the characteristic features of the clustering instability observed in dense media.

The structure of the paper is as follows. In Sec. II a short review of recent results obtained by using kinetic theory methods is presented. The close relationship between the solutions of the Boltzmann and the revised Enskog equations for the homogeneous cooling state is emphasized. It follows that the simulation provides results that, when properly scaled, can be applied to both equations. The simulation results for the velocity distribution function of the homogeneous state are discussed in Sec. III. They are compared with the available kinetic theory predictions and a quite good agreement is observed. In particular, it is found that the first Sonine approximation provides an accurate description over a quite wide range of values of the restitution coefficient. In Sec. IV it is shown that for large enough times the cooling granular medium does not always remain spatially uniform, but, in some cases, spontaneously particles tend to form a cluster surrounded by gas of a much lower density. The results are consistent with a linear hydrodynamics stability theory which finds that instability requires a minimum size of the system when periodic boundary conditions are used $[15,23,24]$. Besides, this minimum size increases as the restitution coefficient approaches unity. Finally, Sec. V provides a short summary and conclusions.

\section{THE HOMOGENEOUS COOLING STATE}

In the revised Enskog theory (RET), the kinetic equation governing the time evolution of the one-particle distribution, $f(\mathbf{r}, \mathbf{v}, t)$, for a system of inelastic smooth spheres with constant coefficient of restitution $\alpha(0<\alpha \leqslant 1)$, is [6]

$$
\left(\partial_{t}+\mathbf{v}_{1} \cdot \nabla_{1}\right) f\left(\mathbf{r}_{1}, \mathbf{v}_{1}, t\right)=\mathcal{J}_{E}\left[\mathbf{r}_{1}, \mathbf{v}_{1}, t \mid f\right],
$$

where $\mathcal{J}_{E}$ is the modified Enskog collision operator,

$$
\begin{aligned}
\mathcal{J}_{E}\left[\mathbf{r}_{1}, \mathbf{v}_{1}, t \mid f\right]= & \sigma^{2} \int d \mathbf{v}_{2} \int d \Omega \Theta(\mathbf{g} \cdot \hat{\boldsymbol{\sigma}})(\mathbf{g} \cdot \hat{\boldsymbol{\sigma}}) \\
& \times\left[\alpha^{-2} g_{e}\left[\mathbf{r}_{1}, \mathbf{r}_{1}-\boldsymbol{\sigma} \mid n\right] f\left(\mathbf{r}_{1}, \mathbf{v}_{1}^{\prime}, t\right)\right. \\
& \times f\left(\mathbf{r}_{1}-\boldsymbol{\sigma}, \mathbf{v}_{2}^{\prime}, t\right)-g_{e}\left[\mathbf{r}_{1}, \mathbf{r}_{1}+\boldsymbol{\sigma} \mid n\right] \\
& \left.\times f\left(\mathbf{r}_{1}, \mathbf{v}_{1}, t\right) f_{1}\left(\mathbf{r}_{1}+\boldsymbol{\sigma}, \mathbf{v}_{2}, t\right)\right] .
\end{aligned}
$$

In the above expression $\sigma$ is the diameter of the spheres, $d \Omega$ is the element of solid angle defined by the unit vector $\hat{\boldsymbol{\sigma}}$ pointing from particle 2 to particle $1, \boldsymbol{\sigma}=\sigma \hat{\boldsymbol{\sigma}}, \Theta$ is the Heaviside step function, $\mathbf{g}=\mathbf{v}_{1}-\mathbf{v}_{2}$, and the primes on the velocities denote scattered values given by

$$
\begin{aligned}
& \mathbf{v}_{1}^{\prime}=\mathbf{v}_{1}-\frac{1}{2} \alpha^{-1}(1+\alpha)(\hat{\boldsymbol{\sigma}} \cdot \mathbf{g}) \hat{\boldsymbol{\sigma}}, \\
& \mathbf{v}_{2}^{\prime}=\mathbf{v}_{2}+\frac{1}{2} \alpha^{-1}(1+\alpha)(\hat{\boldsymbol{\sigma}} \cdot \mathbf{g}) \hat{\boldsymbol{\sigma}} .
\end{aligned}
$$

Therefore, for $\alpha<1$ there is a reduction of the relative velocity along the line of centers so that total momentum is conserved, while kinetic energy is dissipated. Finally, $g_{e}\left[\mathbf{r}_{1}, \mathbf{r}_{2}, t \mid n\right]$ is the equilibrium pair correlation function corresponding to the density field

$$
n(\mathbf{r}, t)=\int d \mathbf{v} f(\mathbf{r}, \mathbf{v}, t)
$$

The RET is a highly nonlinear equation through the dependence of $g_{e}\left[\mathbf{r}_{1}, \mathbf{r}_{2} \mid n\right]$ on $f$. In the low-density limit, the Enskog collision operator reduces to

$$
\begin{aligned}
\mathcal{J}_{B}\left[\mathbf{r}_{1}, \mathbf{v}_{1}, t \mid f\right]= & \sigma^{2} \int d \mathbf{v}_{2} \int d \Omega \Theta(\mathbf{g} \cdot \hat{\boldsymbol{\sigma}})(\mathbf{g} \cdot \hat{\boldsymbol{\sigma}}) \\
& \times\left[\alpha^{-2} f\left(\mathbf{r}_{1}, \mathbf{v}_{1}^{\prime}, t\right) f\left(\mathbf{r}_{1}, \mathbf{v}_{2}^{\prime}, t\right)\right. \\
& \left.-f\left(\mathbf{r}_{1}, \mathbf{v}_{1}, t\right) f_{1}\left(\mathbf{r}_{1}, \mathbf{v}_{2}, t\right)\right],
\end{aligned}
$$

and Eq. (1) becomes the Boltzmann equation. Here we are interested in the homogeneous cooling state (HCS) for which the system is spatially uniform and the dynamics is entirely controlled through the time dependence of the temperature defined by

$$
\frac{3}{2} n k_{B} T=\int d \mathbf{v} \frac{1}{2} m v^{2} f
$$

where $m$ is the mass of the particles and $k_{B}$ the Boltzmann constant. Dimensional analysis and symmetry considerations show that the normal solution of the Enskog equation describing the HCS has the form

$$
f_{H}(\mathbf{v}, t)=n v_{0}^{-3}(t) \phi\left(v / v_{0}(t)\right),
$$

where $v_{0}(t)=\left(2 k_{B} T(t) / m\right)^{1 / 2}$. Besides, the energy balance equation for the HCS is [6]

$$
\partial_{t} T(t)=-\frac{2}{3 n k_{B}}\left(1-\alpha^{2}\right) \omega[T(t)],
$$

with $\omega$ given by 


$$
\omega[T(t)]=\frac{\pi}{16} g_{e}(\sigma) m(n \sigma)^{2} v_{0}^{3}(t) \int d \mathbf{v}_{1} d \mathbf{v}_{2} \phi\left(\mathbf{v}_{1}\right) \phi\left(\mathbf{v}_{2}\right) g^{3} .
$$

Since all the temperature dependence in Eq. (9) is known, it can be easily integrated yielding

$$
T(t)=T(0)\left(1+\frac{t}{t_{0}}\right)^{-2}
$$

with the time constant $t_{0}$ given by

$$
t_{0}^{-1}=\frac{2}{3}\left(1-\alpha^{2}\right) \omega[T(0)]\left[m n v_{0}^{2}(0)\right]^{-1} .
$$

Of course, this equation only holds if the system was already in the HCS at $t=0$. Substitution of Eq. (8) into Eq. (1) and use of Eq. (9) leads to

$$
\left(1-\alpha^{2}\right)\left[\phi(v)+\frac{v}{3} \frac{\partial}{\partial v} \phi(v)\right] W[\phi]=I[v / \phi],
$$

where $W$ and $I$ are functionals of $\phi$ proportional to $\omega$ and the collision term, respectively,

$$
\begin{gathered}
W[\phi]=\frac{\pi}{8} \int d \mathbf{v}_{1} d \mathbf{v}_{2} \phi\left(\mathbf{v}_{1}\right) \phi\left(\mathbf{v}_{2}\right) g^{3}, \\
I\left[v_{1} \mid \phi\right]=\int d \mathbf{v}_{2} \int d \Omega \Theta(\mathbf{g} \cdot \boldsymbol{\sigma})(\mathbf{g} \cdot \hat{\boldsymbol{\sigma}}) \\
\quad \times\left[\alpha^{-2} \phi\left(v_{1}^{\prime}\right) \phi\left(v_{2}^{\prime}\right)-\phi\left(v_{1}\right) \phi\left(v_{2}\right)\right] .
\end{gathered}
$$

Let us notice that Eq. (13) does not contain the equilibrium correlation function and remains unchanged in the lowdensity limit. The only difference between the solutions of the Enskog equation and the Boltzmann equation describing the HCS is a constant factor in the expression of $\omega[T(t)]$, i.e., the rate of change of the temperature from both equations differs by a constant, namely, the equilibrium pair correlation function of two spheres at contact. Therefore, when properly scaled, the solutions of both the Enskog and the Boltzmann equations for the HCS coincide.

Although Eq. (13) is a closed equation for $\phi$, solving it is not an easy task, and only approximated solutions have been found up to now. In particular, in Refs. [9] and [14] the function $\phi$ is expanded in a series of Sonine polynomials $S_{1 / 2}^{(j)}$

$$
\phi(v)=\phi^{(0)}(v) \sum_{j=0}^{\infty} a_{j} S_{1 / 2}^{(j)}\left(v^{2}\right)
$$

where $\phi^{(0)}(v)=\pi^{-3 / 2} e^{-v^{2}}$ corresponds to the MaxwellBoltzmann distribution with a time-dependent temperature and the Sonine polynomials are defined by [13]

$$
S_{l}^{j}(x)=\sum_{p=0}^{j} \frac{\Gamma(j+l+1)}{(j-p) ! p ! \Gamma(p+l+1)}(-x)^{p} .
$$

From the normalization condition and the definition of temperature Eq. (7) it follows that $a_{0}=1$ and $a_{1}=0$. Besides, if contributions to the moments nonlinear in $a_{2}$ are neglected (first Enskog approximation), it is found that [14,25]

$$
a_{2}=\frac{16(1-\alpha)\left(1-2 \alpha^{2}\right)}{81-17 \alpha+30 \alpha^{2}-30 \alpha^{3}}
$$

From the expression of $S_{1 / 2}^{(2)}$ and Eq. (8) it is easily seen that

$$
\frac{3}{5} \frac{\left\langle v^{4}\right\rangle_{H}}{\left\langle v^{2}\right\rangle_{H}^{2}}=1+a_{2},
$$

where

$$
\left\langle v^{l}\right\rangle_{H}=\frac{1}{n} \int d \mathbf{v} v^{l} f_{H}(\mathbf{v}, t),
$$

are the velocity moments. The fact that the absolute value of the right hand side of Eq. (18) is bounded by 0.2 has been used to justify the approximation of the homogeneous solutions of the Enskog and Boltzmann equations by the Maxwell-Boltzmann distribution. Of course, this argument is of limited value, not only because it is restricted to the first three terms of the Sonine expansion of the distribution function, but also because the own coefficient $a_{2}$ has been calculated in the first Enskog approximation.

\section{SIMULATION RESULTS FOR THE HOMOGENEOUS COOLING STATE}

The direct simulation Monte Carlo method [21] was devised in order to mimic the dynamics described by the Boltzmann equation, and has been successfully applied to a wide range of phenomena in rarefied gases. Since the method has been extensively discussed in Refs. [21] and [22], it will not be described here. The only modification needed to apply it to a gas of inelastic hard spheres is given by the expression of the postcollision velocities,

$$
\begin{aligned}
& \mathbf{v}_{1}^{*}=\mathbf{v}_{1}-\frac{1+\alpha}{2}(\mathbf{g} \cdot \hat{\boldsymbol{\sigma}}) \hat{\boldsymbol{\sigma}}, \\
& \mathbf{v}_{2}^{*}=\mathbf{v}_{2}+\frac{1+\alpha}{2}(\mathbf{g} \cdot \hat{\boldsymbol{\sigma}}) \hat{\boldsymbol{\sigma}} .
\end{aligned}
$$

In our simulations we have used the so-called "timecounter" method, which involves the calculation of representative collisions and, at each collision, the time counter of the cell in which the collision takes place is advanced by an amount appropriate to the collision. Although there exist other alternative schemes which are more accurate for extreme nonequilibrium situations, such as the NTC method [22], they are equivalent to the time-counter method for our present purposes.

The system we have considered consists of $N$ particles enclosed between two plates perpendicular to the $y$ axis separated by a distance $L$. In the simulation the system is split into $N_{c}$ cells which are layers of the same width parallel to the plates. All the cells have initially the same number of particles, i.e., the initial density is uniform. Periodic boundary conditions are applied in the $y$ direction. Besides, we have used reduced units defined by $m=1, k_{B} T(0)=1 / 2$, and $\lambda=1$, where $\lambda \equiv\left(\sqrt{2} \pi n \sigma^{2}\right)^{-1}$ is the mean free path. 


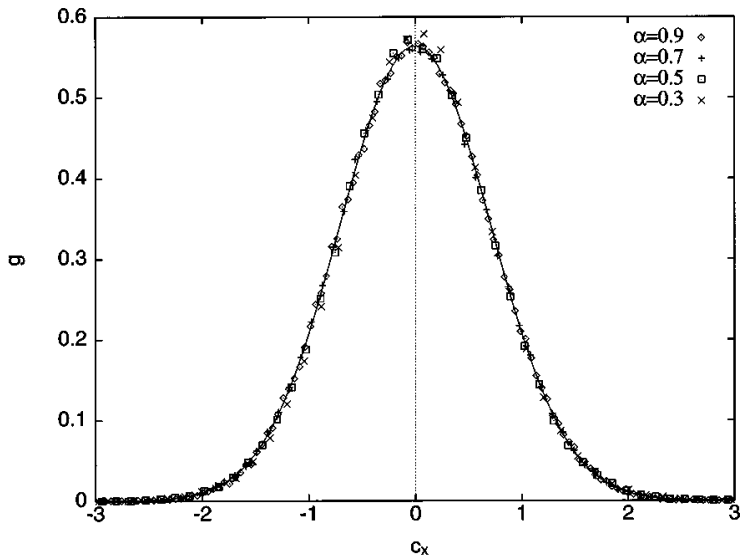

FIG. 1. Scaled marginal velocity distribution function for the HCS as a function of the reduced velocity for several values of the restitution coefficient.

As a first test of whether the simulation reproduces the HCS, we have checked the scaling property given by Eq. (8). In Fig. 1 we have plotted

$$
g\left(v_{x}, t\right)=n^{-1} v_{0}^{3}(t) \int d v_{y} d v_{z} f(\mathbf{v}, t),
$$

as a function of $c_{x} \equiv v_{x} / v_{0}(t)$ for several values of the restitution coefficient, namely, $\alpha=0.3,0.5,0.7$, and 0.9 . Of course, in the simulation the right hand side of Eq. (22) is computed by discretizing the velocity space and counting the number of particles with velocities inside each of the intervals. The time-dependent temperature has been measured using its definition, Eq. (7). The numerical values of the technical parameters of the simulation are $N=1000, L=10 \lambda$, and $N_{c}=20$. Besides, the time step over which it is assumed that free motion and collisions are uncoupled has been taken $\Delta t=0.1$, and the reported distributions correspond to $t=25$, having been averaged over 150 trajectories. The statistical

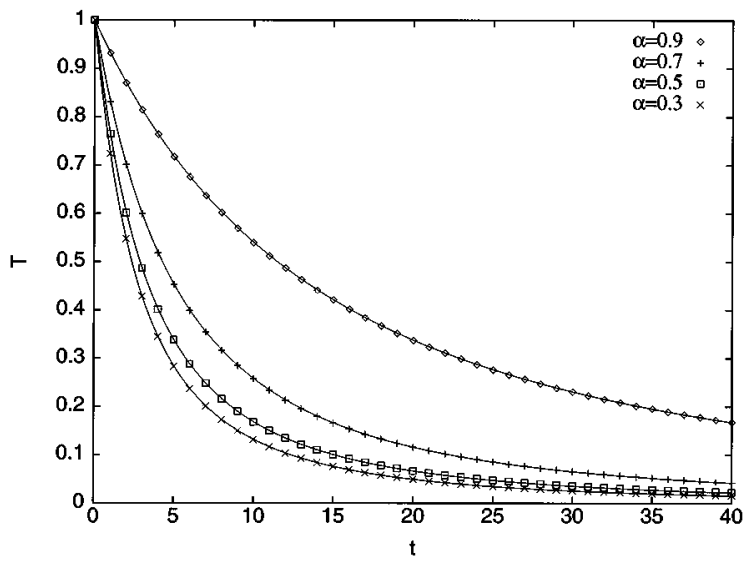

FIG. 2. Time evolution of the temperature for several values of the restitution coefficient. In all cases, the initial state was homogeneous with a Maxwellian velocity distribution. The symbols are results from the simulation, and the solid line is the best fit to Eq. (11).

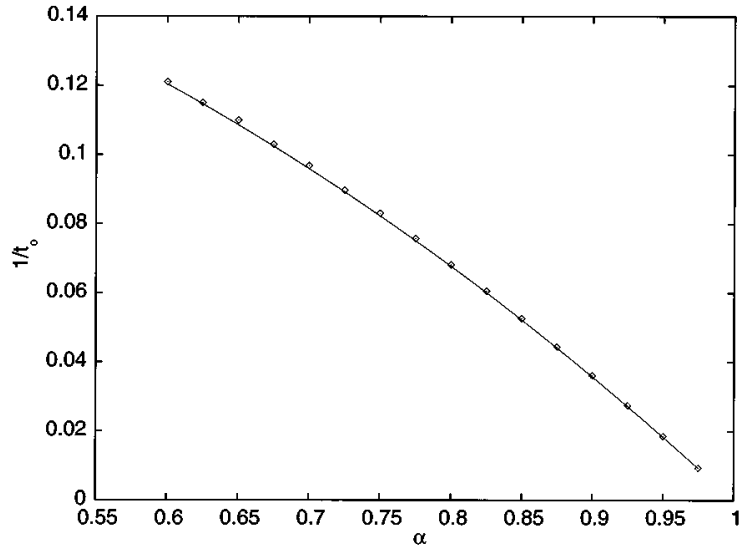

FIG. 3. Values of the parameter $t_{o}$ defined in Eq. (11) as a function of the restitution coefficient. The points have been obtained from the fitting in Fig. 2, while the solid line corresponds to Eq. (24).

uncertainties are smaller than the symbols used to represent the data. The initial distribution has been in all cases a Maxwellian with a temperature $T(0)$. From Fig. 1 it is seen that the scaling law is verified by the simulation data. Furthermore, over the used scale, the data are perfectly fitted by the Maxwell-Boltzmann distribution $\phi_{x}^{(0)}\left(c_{x}\right)=\pi^{-1 / 2} \exp \left(-c_{x}^{2}\right)$. Similar results are obtained for the reduced distributions of the other two components of the velocity $v_{y}$ and $v_{z}$. Therefore, if deviations from a Gaussian distribution are to be observed, a much finer scale must be used. This will be done later on.

The time evolution of the temperature for each of the values of $\alpha$ given above is shown in Fig. 2. Also plotted are the best fits to the right hand side of Eq. (11) with $t_{0}$ an adjustable parameter. Again an excellent agreement is obtained. Besides, Fig. 3 shows that the values of $t_{0}$ obtained in this way are reproduced, within the error bars, by Eq. (12)

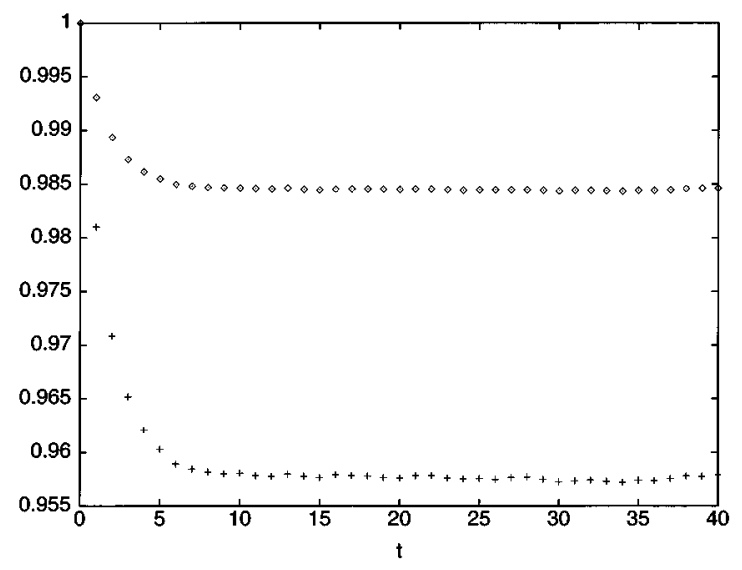

FIG. 4. Time evolution of the fourth (diamonds) and sixth (crosses) velocity moments for $\alpha=0.9$. The initial state is homogeneous with a Maxwellian distribution of velocities. 


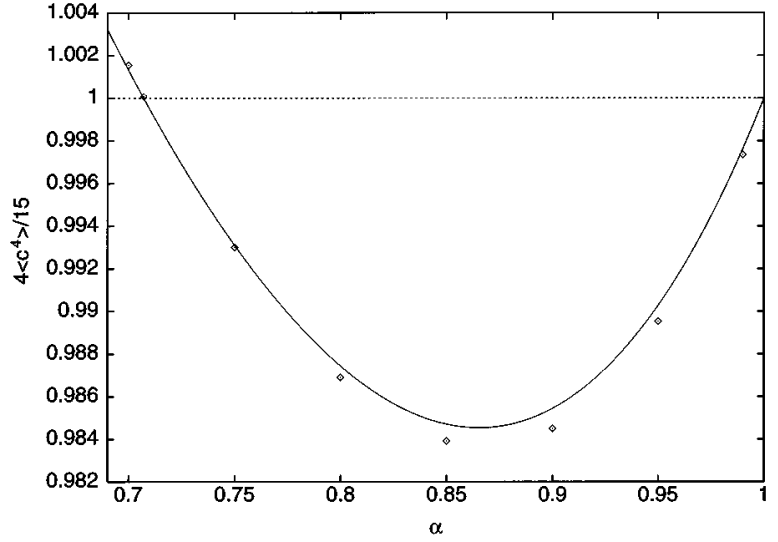

FIG. 5. Comparison of the value of the fourth moment in the HCS as obtained from the simulation (diamonds) and the theoretical prediction obtained in the first Enskog approximation (solid line).

with $\omega[T(0)]$ computed from the initial Maxwell distribution, i.e.,

$$
\begin{gathered}
\omega[T(0)]=\frac{\pi}{16}(n \sigma)^{2} v_{0}^{3}(0) \int d \mathbf{v}_{1} d \mathbf{v}_{2} \phi^{(0)}\left(v_{1}\right) \phi^{(0)}\left(v_{2}\right) \\
=2\left(\frac{\pi}{m}\right)^{1 / 2}(n \sigma)^{2}\left[k_{B} T(0)\right]^{3 / 2} \\
t_{0}^{-1}=\frac{1}{6}\left(1-\alpha^{2}\right) \nu(0)
\end{gathered}
$$

where $\nu=4 \sigma^{2} n\left(\pi k_{B} T / m\right)^{1 / 2}$ is the collision frequency. Notice that, since Eq. (11) only holds in the HCS, this result confirms the accuracy of the Maxwell-Boltzmann approximation to describe the time evolution of the temperature in this state. To investigate deviations from that approximation we have studied the forth and sixth velocity moments. The details of the simulations are the same as described above, but now in order to improve the statistical accuracy the number of trajectories has been largely increased. The results we will discuss in the remainder of this section have been averaged over $10^{5}$ trajectories.

In Fig. 4 we present a typical time evolution of the ratios between the moments obtained from the simulation and those corresponding to a Maxwell-Boltzmann distribution.

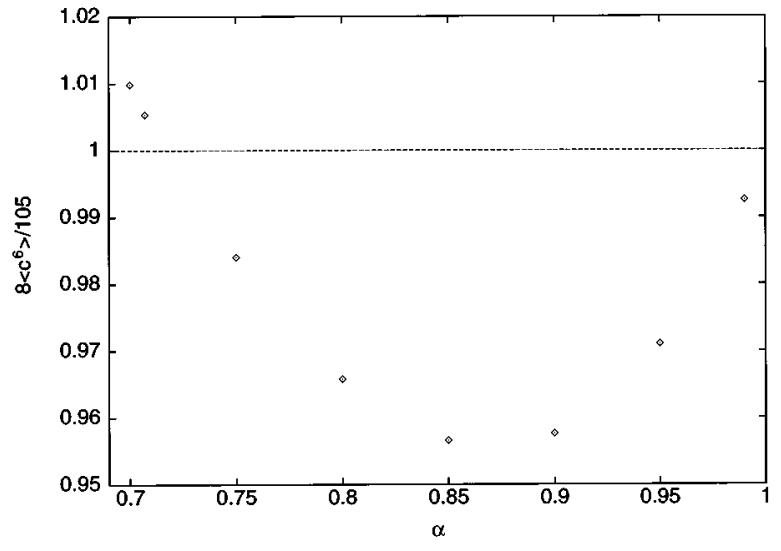

FIG. 6. Values of the sixth velocity moments in the HCS as a function of the restitution coefficient.

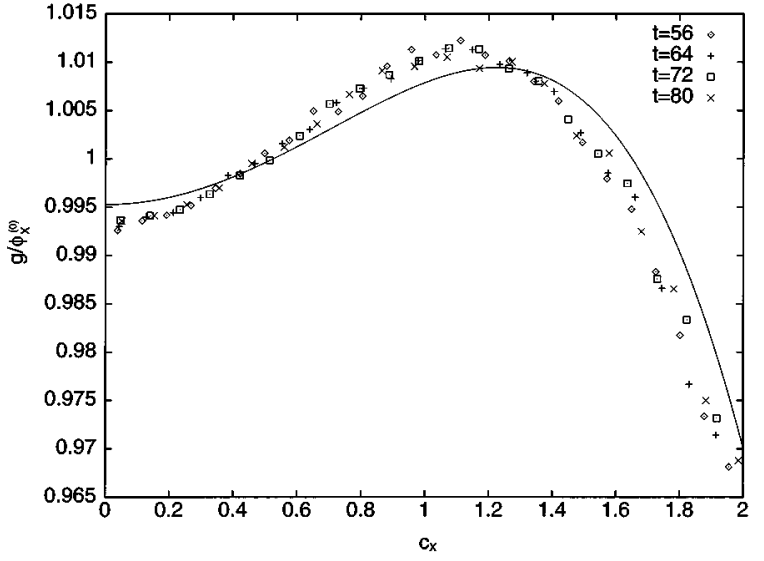

FIG. 7. Scaled marginal velocity distribution function for the HCS for $\alpha=0.8$. The solid line corresponds to Eq. (25).

This is defined with the actual temperature of the system obtained from the simulation. Therefore, what is being measured are deviations of the velocity distribution of the system from a Gaussian, but not a direct estimation of the accuracy of the Maxwell-Boltzmann approximation, in which the temperature is consistently computed in the same approximation, i.e., using Eqs. (11) and (24). When this is done, the deviations of the ratios from unity increase by a factor which is roughly of the order of 2. From Fig. 4 it is seen that, after a transient initial period of time, the ratios reach quite steady values, then providing accurate quantitative measures of the non-Gaussianity of the distribution function of the HCS. The displayed curves correspond to $\alpha=0.9$, but a similar behavior is obtained for other values of the restitution coefficient. The only relevant qualitative change is that for $\alpha$ smaller than a given value to be specified below, the ratio of the two fourth moments increases during the transient regime and the stationary value is larger than unity. The same change occurs to the ratio of the sixth moments.

The results of the simulation for the fourth moments in the HCS can be compared with the theoretical prediction given by Eq. (19). The agreement is quite good as shown in Fig. 5, implying the accuracy of the expression for $a_{2}$ obtained in the first Enskog approximation. In particular, according to Eq. (19), the fourth moment of the HCS coincides

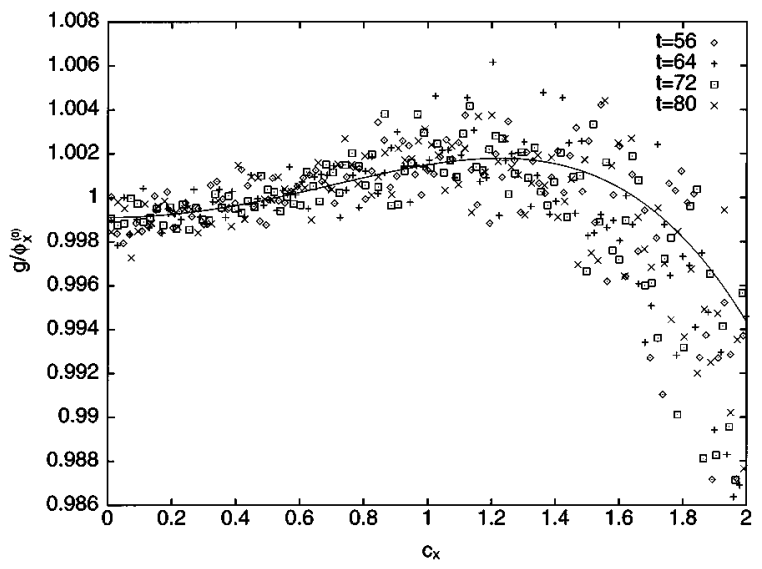

FIG. 8. The same as Fig. 7 but for $\alpha=0.99$. 

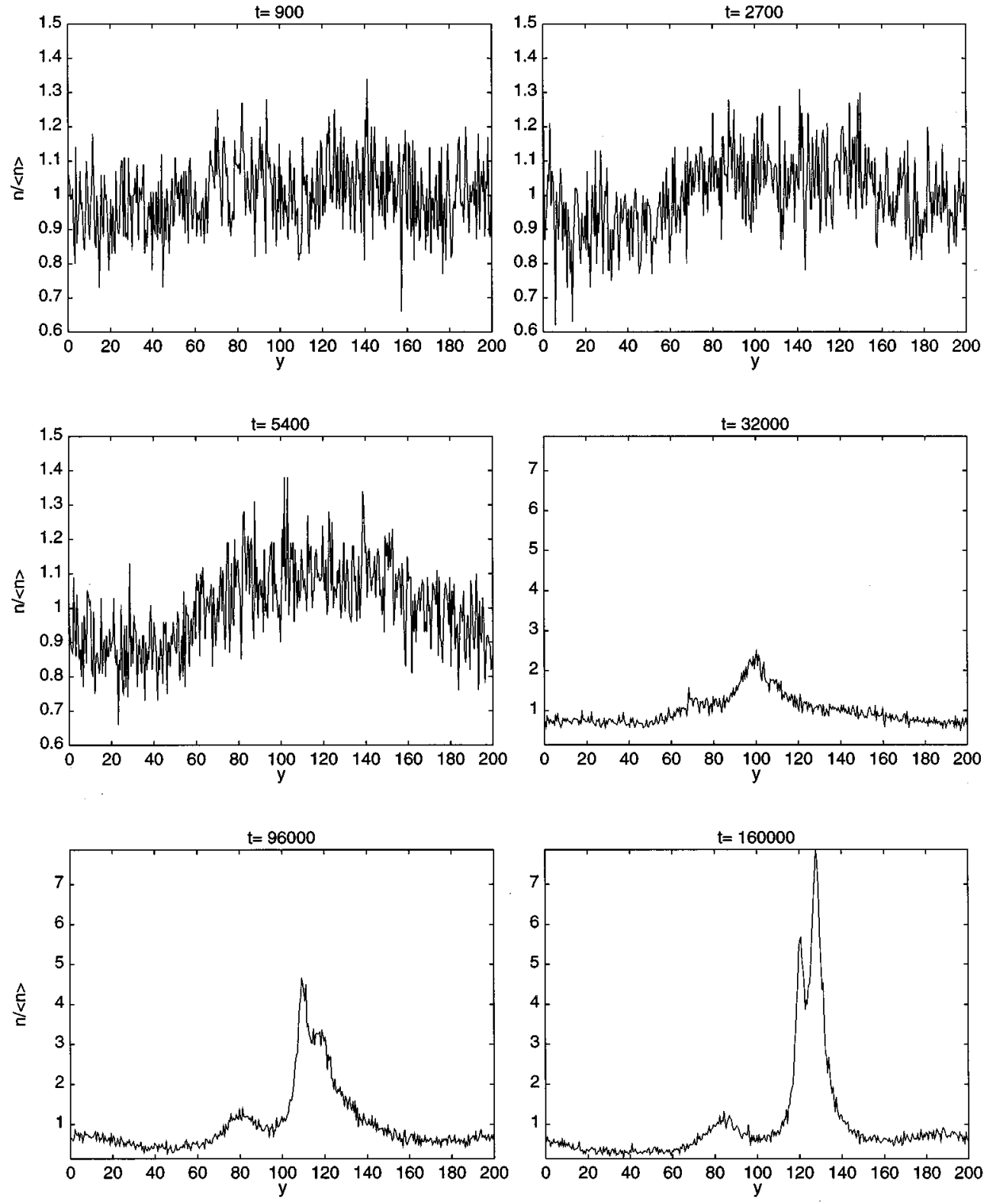

FIG. 9. Time evolution of the density profile along the $Y$ direction for an initially homogeneous system with $\alpha=0.95$ and $L=20$.

with that of the Maxwellian for $\alpha=1 / \sqrt{2}$, which is very close to the value estimated from the simulation. In Fig. 6 we have plotted the results for the sixth moment, again divided by the Maxwellian value. As expected, the discrepancies are now larger, although the curve has a similar shape. We are not aware of any theoretical calculations of this moment to use for a comparison.

Given that Eq. (19) provides a good estimation for the coefficient $a_{2}$, it is interesting to determine whether the second Sonine approximation is also a good approximation for the distribution function of the HCS. Figures 7 and 8 depict the normalized distribution $g\left(c_{x}, t\right) / \phi_{x}^{(0)}\left(c_{x}\right)$ as a function of $c_{x}$ for $\alpha=0.8$ and 0.99 , respectively. The points correspond to simulation results obtained at four different times. Each of them has been averaged over $10^{5}$ trajectories. The solid line is Eq. (16) with the series truncated at $j=2$, i.e.,

$$
\frac{\phi_{x}\left(c_{x}\right)}{\phi_{x}^{0}\left(c_{x}\right)}=1+a_{2} S_{-1 / 2}^{(2)}\left(c_{x}^{2}\right)=1+\frac{a_{2}}{2}\left(c_{x}^{4}-3 c_{x}^{2}+\frac{3}{4}\right)
$$

with $a_{2}$ given by Eq. (19). Notice the different scales used in both figures. The agreement is again excellent, especially taking into account the very small discrepancies we are measuring.

\section{CLUSTERING INSTABILITY}

The focus of this section is the study of the stability of the HCS using the direct simulation Monte Carlo method. It must be noticed that the results reported in this section can not be directly translated to a dense gas described by the Enskog equation, since the equivalence between this equa- 
tion and the Boltzmann equation discussed in Sec. II is restricted to the HCS, but it fails as long as deviations from this state are considered. We were interested in whether the cluster instability which has been observed in molecular dynamics simulations of granular flows in one [16-18] and two dimensions [19] is also present in the Boltzmann equation. Molecular dynamics simulations show that the granular medium becomes spontaneously nonuniform and there is a tendency of particles to form clusters or "inelastic microstructures" surrounded by low-density regions. This tendency increases with the inelasticity of the particles. In some cases the clusters develop a finite time singularity referred to as "inelastic collapse" whereby most of the particles become concentrated at a point cluster. In inelastic collapse a group of particles collide infinitely often in a finite time so that the spacing between particles in this group becomes zero. On the other hand, in the cluster instability particles in the same cluster are close together, but not in contact. While the clustering instability is of hydrodynamic origin, in the sense that it is predicted by an hydrodynamic description of the system [20], the inelastic collapse is of a local nature and, therefore, cannot be captured by a hydrodynamic description.

A very significant result from linear hydrodynamic stability theory is that the instability of the HCS is confined to perturbations with large enough wavelength $[23,24,15]$. More precisely, for each value of the restitution coefficient $\alpha$ there is a critical value $k^{*}(\alpha)$ such that perturbations with wave number $k>k^{*}(\alpha)$ are always stable. In a given system, the smallest wave number $k_{m}$ allowed for a perturbation or fluctuation can be estimated as $2 \pi / L$, where $L$ is the parameter length characterizing the size of the system. It is clear that for the system geometry we used in our simulations, $L$ coincides with the distance between the two plates perpendicular to the $y$ axis, since no spatial dependence in the $x$ and $z$ directions is considered. If $k_{m}>k^{*}(\alpha)$ the HCS will be asymptotically stable because the fluctuations that would lead to the development of instabilities are not compatible with the boundary conditions.

In Fig. 9 we present the time evolution of the density profile along the $y$ direction for one realization of an initially homogeneous system with $\alpha=0.95$ and $L=200$. The number of particles is $N=4 \times 10^{4}$. In spite of the noise inherent to the fact that no average is being used, the spontaneous formation of a wave perturbation with wavelength $L$ clearly shows up for times about 2700. Similar results were obtained for all the realizations we have simulated. As the amplitude of the perturbation grows no oscillatory behavior is observed. Besides, the simulation results show that the pressure of the system remains approximately uniform and, consequently, the temperature also shows a wave-shaped perturbation. Let us notice that the instability appears for times much larger than those used in the preceding section to investigate the velocity distribution of the HCS. The process ends up with the formation of a cluster with a high concentration of particles surrounded by a gas of much lower density. This can be identified with the cluster instability observed in molecular dynamics simulations. Nevertheless, it must be kept in mind that what we are studying are solutions of the Boltzmann equation and, therefore, their validity is restricted to low density gases. As a consequence, one should not expect our results to give a quantitative description of the nature of the cluster as observed in molecular dynamics simulations. For the same reason, the direct simulation Monte Carlo method cannot lead to inelastic collapse. By definition, velocities of colliding particles are not correlated in this simulation technique, and velocity correlations are one of the main signatures of inelastic collapse. The relevant conclusion emerging from Fig. 9 is that the Boltzmann equation contains the physical mechanisms which are responsible for the appearance of the "seed" of the cluster instability.

To investigate whether there is a critical value $k^{*}$ as discussed above, we have simulated a system with the same value $\alpha=0.95$ of the restitution coefficient but with $L=40$. In this case, no instability was observed even for times much larger than those considered in the simulation in Fig. 9. As a stronger test, we introduced an initial perturbation of the form $n(y, 0)=n_{0}(1+0.3 \sin k y)$, where $k=2 \pi / L$. The perturbation decayed quite fast in all the realizations. We conclude that the system is stable, supporting the dependence of stability on the size of the system.

Finally, in order to check the dependence of $k^{*}$ on $\alpha$ we considered a system of width $L=40$ and $\alpha=0.8$. Again, a spontaneous wave-shaped perturbation develops quite soon and, at later times, the particles concentrate in a narrow layer forming a cluster. In fact, the effect is faster and stronger than in the case reported in Fig. 9. This was to be expected since now the restitution coefficient is smaller. In conclusion, the simulation results fully confirm that the Boltzmann equation is qualitatively consistent with the scenario of stability which has been derived from hydrodynamic theories and observed in molecular dynamics simulations of inelastic dense fluids.

\section{CONCLUSIONS}

It has been shown that the direct simulation Monte Carlo method provides a useful tool to study low-density inelastic fluids. When applied to the homogeneous cooling state, it allows a quite accurate measurement of the velocity distribution function for arbitrary values of the restitution coefficient. The results are in good agreement with the analytical solution of the Boltzmann equation in the first Enskog approximation, specially for values of the restitution coefficient not too small. In particular, an excellent agreement is found for the fourth velocity moment. The same tool can be used to investigate other states of the granular medium, even far from equilibrium.

We have also studied the stability of the uniform state by analyzing both the appearance of spontaneous inhomogeneities in the system and the response to an initial sinusoidal perturbation of small amplitude. Large enough systems are unstable and develop inhomogeneities which for large times lead to the formation of particle clumps. Since the Boltzmann equation, and consequently the simulation, neglects correlations between colliding particles, it cannot correctly describe the system in the long time limit, even if the initial density average was very low. Nevertheless, it must qualitatively capture some of the characteristic physical features which are precursors of the cluster state. Therefore, a careful analysis of the instability of the Boltzmann equation, combining both theory and simulation, seems to be worthwhile. We emphasize that the conclusion that the homoge- 
neous fluid is unstable in the low-density limit is not restricted in any way.

Of course, a better understanding of the initial stage of the cluster formation may be obtained by using the modified Enskog equation discussed in Sec. II, since it incorporates spatial correlations between particles. Very recently, the direct simulation Monte Carlo method has been extended to this equation, and some initial promising results for the uniform shear flow state of a normal fluid have been obtained [26]. We plan to study the development of the clustering instability in the modified Enskog equation by using this simulation technique to analyze the influence of spatial correlations in the formation of clusters.

\section{ACKNOWLEDGMENTS}

This research was partially supported by Grant No. PB960534 from the Dirección General de Investigación Científica y Técnica (Spain). We are very grateful to Dr. M. H. Ernst for enlightening discussions and also for providing us with results prior to publication.
[1] C.S. Campbell, Annu. Rev. Fluid Mech. 22, 57 (1990).

[2] J.T. Jenkins and S.B. Savage, J. Fluid Mech. 130, 187 (1983).

[3] P.K. Haff, J. Fluid Mech. 134, 401 (1983).

[4] C. Lun, S. Savage, D. Jeffrey, and N. Chepurniy, J. Fluid Mech. 140, 223 (1984).

[5] J.T. Jenkins and M.W. Richman, Arch. Rat. Mech. 87, 355 (1985); Phys. Fluids 28, 3485 (1985).

[6] J.J. Brey, J.W. Dufty, and A. Santos, J. Stat. Phys. (to be published).

[7] C.K.K. Lun and S.B. Savage, J. Appl. Mech. 54, 47 (1987).

[8] C.K.K. Lun, J. Fluid Mech. 233, 539 (1991).

[9] A. Goldshtein and M. Shapiro, J. Fluid Mech. 282, 75 (1995).

[10] M. Farrell, C.K.K. Lun, and S.B. Savage, Acta Mech. 63, 45 (1986).

[11] T.J. Jenkins and F. Mancini, Phys. Fluids A 1, 2050 (1989).

[12] P. Zamankhan, Phys. Rev. E 52, 4877 (1995).

[13] P. Resibois and M. de Leener, Classical Kinetic Theory of Fluids (Wiley, New York, 1977).

[14] T.P.C. van Noije, R. Brito, and M.H. Ernst (unpublished).

[15] J.J. Brey, F. Moreno, and J.W. Dufty, Phys. Rev. E 54, 445 (1996).
[16] B. Bernu and R. Mazighi, J. Phys. A 23, 5745 (1990).

[17] S. McNamara and W.R. Young, Phys. Fluids A 4, 496 (1992); 5, 34 (1993).

[18] N. Sela and I. Goldhirsch, Phys. Fluids 7, 507 (1995).

[19] S. McNamara and W.R. Young, Phys. Rev. E 50, R28 (1994).

[20] I. Goldhirsch and G. Zanetti, Phys. Rev. Lett. 701619 (1993).

[21] G. Bird, Molecular Gas Dynamics (Oxford University Press, New York, 1976).

[22] G. Bird, Molecular Gas Dynamics and the Direct Simulation of Gas Flows (Clarendon Press, Oxford, 1994).

[23] M. Babic, in Advances in Micromechanics of Granular Materials, edited by H.H. Shen, M. Satake, M. Mehradabi, C.S. Chang, and C.S. Campbell (Elsevier, Amsterdam, 1992), p. 291.

[24] S. McNamara, Phys. Fluids A 5, 3056 (1993).

[25] The coefficient $a_{2}$ was also calculated by Goldstein and Shapiro in Ref. [9] using the same approximation as in Ref. [14], but there is an error in their calculations.

[26] J.M. Montanero and A. Santos, Phys. Rev. E 54, 438 (1996). 